Z. klin. Chem. u. klin. Biochem.

7. Jg., S. 480-485, September 1959

\title{
Zur Messung der in vivo- und in vitro-Reaktivierbarkeit alkylphosphatvergifteter Serumcholinesterase durch 2-PAM und Toxogonin mit verschiedenen Substraten
}

\author{
Von Marika Geldmacher-v. Mallinckrodt, H. J. Urbach, H. KitTiel und H. H. Lindorf \\ Aus dem Institut für gericbtliche Medizin und Kriminalistik der Universität Erlangen-Nürnberg \\ (Direktor: Prof. Dr. Dr. E. Weinig) \\ (Eingegangen am 31. März 1969)
}

\begin{abstract}
Zur Messung derCholinesteraseaktivität in Gegenwart von 2-PAM und Toxogonin sind echte Ester wie Acetylcholin, Butyrylcholin und Benzoylcholin geeignete Substrate. Substrate mit Säureanhydridcharakter wie Acetylthiocholin, Butyrylthiocholin, o-Nitrophenylbutyrat, Indoxylacetat, Phenylbenzoat und $\alpha$-Naphtylacetat werden durch die beiden Oxime gespalten. Das Ausmaß der Spaltung ist jedoch von der Oximmenge im Ansatz abhängig. Nach Gaben von 2-PAM und Toxogonin in dẹn empfohlenen therapeutischen Mengen sind deshalb Verfahren, die Acetylthiocholin, Butyrylthiocholin und o-Nitrophenylbutyrat verwenden, trotzdem brauchbar.
\end{abstract}

Measurement of the in vivo and in vitro reactivation of alkyl phosphate-poisoned serum cbolinesterase by 2-PAM and toxogonin in the presence of different substrates

True esters, like acetyl choline, butyrylcholine and benzoyl choline are suitable substrates for the measurement of cholinesterase activity in the presence of 2-PAM and toxogonin. Substrates with acid anhydride chatacter, likeacetylthiocholine, butyrylthiocholine, o-nitrophenyl butyrate, indoxyl acetate, phenyl butyrate and $\alpha$-naphtyl acetate are all cleaved by the two oximes. The extent of the cleavage is, however, proportional to the concentration of the oxime. Therefore, following the administration of 2-PAM and toxogonin in the recommended therapeutic amounts, methods that employ acetylthiocholine, butyrylthiocholine and o-nitrophenyl butyrate can, nevertheless, be used.

Oxime wie 2-PAM (2-Pyridinaldoximmethyljodid) und Toxogonin (Bis-(4-hydroxyiminomethyl-1-pyridiniummethyl)-ätherdichlorid) werden zur Therapie von Vergiftungen mit Alkylphosphaten, insbesondere $\mathrm{E} 605$ eingesetzt. Dabei interessierte in bestimmten Fällen eine Kontrolle des Therapieerfolges durch Messung der Serumcholinesterase-Aktivität nach der Oximgabe. Zur grundsätzlichen Entscheidung, ob ein Oxim zur Reaktivierung eines phosphorylierten Enzyms in der Lage ist, wurde von einigen Autoren der in vitro-Test herangezogen. Bei klinischen Fällen haben z. B. HeirmanN und Felgenhauer (1) versucht, vor der therapeutischen Anwendung die Wirksamkeit des Reaktivators an der in vivo alkylphosphatvergifteten Serumcholinesterase der Patienten durch in vitro-Inkubation mit Toxogonin $\mathrm{zu}$ testen.

Auch forensisch spielt die Bestimmung der Cholinesterase-Aktivität im Serum bei der Beurteilung von Vergiftungsfällen eine Rolle. Auf Grund der starken individuellen Unterschiede in der Aktivität des normalen Enzyms, des Vorkommens atypischer Serumcholinesterasen mit $z$. T. sehr niedriger oder fehlender Aktivität, des Einflusses von Krankheiten sowie der Möglichkeit einer Cholinesterase-Blockierung nicht nur durch Alkylphosphate, sondern auch durch Carbamate und viele Medikamente haben FrIEDBERG und SAKAI (2) für forensische Zwecke ein Verfahren zur in vitro-Reaktivierung alkylphosphatvergifteter Cholinesterasen mit 2-PAM ausgearbeitet. Sie empfehlen, mit einer beliebigen Methode die Aktivität ohne und mit 2-PAM-Zusatz zu prüfen. Ergebe sich durch PAM eine Aktivitätssteigerung, so liege mit Sicherheit eine Vergiftung mit einem organischen Phosphorsäureester vor. Die Autoren selbst verwendeten zur Aktivitätsbestimmung die manometrische Methode von AMmon (3) mit Acet; 1 cholin als Substrat.

HeitmanN und Felgenhauer (1) wiesen kürzlich darauf hin, daß Toxogonin eine ,esterolytische" Wirkung besitzt, wodurch es in der Lage ist, Substrate wie Acetylthiocholin, Butyrylthiocholin und $\alpha$-Naphthylacetat zu spalten und damit eine stark reaktivierte Cholinesterase vorzutäuschen. NENNER und ERDMANN (4) haben dies für Acetylthiocholin bestätigt.

Schon BERgNer und O'NeIly (5) hatten gezeigt, daß Oxime wie 2-PAM, 4-PAM und DAM .(Diacetylmonoxim) Acetyl- und Butyrylthiocholin im schwach alkalischen Milieu unter Freisetzung von Thiocholin spalten. Dieser Effekt war bei pH 5 praktisch nicht mehr $z u$ beobachten, was die Autoren darauf zurückführen, $\mathrm{da}$ nur bei höheren $\mathrm{pH}-$ Werten das Gleichgewicht zugunsten des allein in Reaktion tretenden Oximations verschoben ist. Bei Durchführung der enzymatischen Spaltung bei pH 5,3 konnten sie eine Störung infolge Oximwirkung ausschalten.

Einen ähnlichen Effekt auf die beiden Thioester wie die genannten Oxime haben auch Imidazol und Cystein (6). Wir selbst (7) konnten die Spaltung von o-Nitrophenylbutyrat durch 2-PAM und Toxogonin zeigen.

Damit ist grundsätzlich die Frage zu klären, welche Substrate für die Prüfung des klinischën Erfolges von Oximgaben bzw. für in vitro-Inkubation geeignet sind, und welche für solche Untersuchungen ausscheiden.

In einer früheren Atbeit (7) war die Vermutung ausgesprochen worden, daß ähnlich wie o-Nitrophenylbutyrat auch andere Substrate mit Säureanhydrid-Charakter durch Oxime gespalten werden, ;während dies bei echten Estern wie z. B. Acetylcholin nicht der Fall ist. Dafür sprechen die Ergebnisse von FrIEDberg und SAKaI (2) 
sowie Sucker (8) mit Acetylcholin und 2-PAM unter Verwendung der manometrischen Methode bzw. eines kolorimetrischen und potentiometrischen Verfahrens, sowie von NENNER und ERDMANN (4) mit Acetylcholin und Toxogonin bei Anwendung einer titrimetrischen Methode.

Wir führten deshalb mit verschiedenen für die Bestimmung der Cholinesterase-Aktivität in der Literatur angegebenen Substraten Versuche durch, in denen wir neben unvergiftetem menschlichem Serum auch 2-PAM und Toxogonin dem Ansatz zufügten. Damit.sind nun die folgenden Verbindungen auf ihre Reaktion mit den beiden genannten Oximen geprüft:

Acetylthiocholin und 2-PAM

Acetylthiocholin und Toxogonin (vgl. auch (1), (4))

Butyrylthiocholin und 2-PAM

Butyrylthiocholin und Toxogonin (vgl. auch (1))

o-Nitrophenylbutyrat und 2-PAM (vgl. auch (7))

o-Nitrophenylbutyrat und Toxogonin (vgl. auch (17))

Indoxylacetat und 2-PAM

Indoxylacetat und Toxogonin

Phenylbenzoat und 2-PAM

Phenylbenzoat und Toxogonin

$\alpha$-Naphtylacetat und 2-PAM

$\alpha$-Naphtylacetat und Toxogonin (vgl. auch (1))

Acetylcholin und 2-PAM (vgl. auch (2), (8))

Acetylcholin und Toxogonin (vgl. auch (4))

Butyrylcholin und 2-PAM

Butyrylcholin und Toxogonin

Benzoylcholin und 2-PAM

Benzoylcholin und Toxogonin.

\section{Methodik und Ergebnisse}

Für die Untersuchungen verwendeten wir reines 2-PAM in Form des Jodids und Toxogonin (Obidoximchlorid). Die Präparate stellten Gemische der syn- und anti-Form in wechselnder Zusammensetzung dar. Sie wurden den Ansätzen in Mengen zugesetzt, die im Falle einer Substratspaltung etwa gleichen Umsatz ergaben wie der jeweilige Serumzusatz. Zum Vergleich wurden deshalb stets normale Serumproben mitgeführt. Die Versuchsbedingungen entsprachen den von den einzelnen $\mathrm{Au}-$ toren angegebenen.

Bei einigen Substraten wurden auch Vielfache der ebengenannten Oximmengen geprüft, ưm einen Anhaltspunkt für die Abhängigkeit der Substratspaltung von der Oximmenge zu gewinnen.

Mit Ausnahme von o-Nitrophenylbutyrat zeigte die Substratspaltung durch die beiden Oxime schon kurz nach dem Starten der Reaktion während des Beobachtungszeitraumes einen linearen Verlauf.

Entsprechendes galt für die Spaltung aller Substrate durch die Serumcholinesterasen. Um eine bessere Vergleichsmöglichkeit zu erhalten, wurde deshalb im Sinne eines „kinetischen Tests" (vgl. KNEDEI und BörTGER (9)) nur der lineare Anteil der Beziehung zwischen Substratspaltung (wiedergegeben durch die Extinktions- zunahme bzw. die $\mathrm{CO}_{2}$-Entwicklung) und Zeit wiedergegeben, der ein $\mathrm{Maß}$ für die Geschwindigkeit der Hauptreaktion darstellt. Lediglich bei o-Nitrophenylbutyrat ist auch der Anfangsteil der Reaktion wiedergegeben.

Die Ergebnisse wurden graphisch dargestellt, wobei jeder Punkt den Mittelwert aus mindestens drei Einzelbestimmungen darstellt.

Acetyltbiocholin nach WeBEr (10).

Reaktionsbedingungen:

0,05M Tris-Puffer: $\mathrm{pH} 7,4$;

Tris-Puffer-DTNB-(5,5-Dithiobis-2-nitrobenzoesäure)-Lösung:

$20 \mathrm{mg}$ DTNB in $200 \mathrm{~m} /$ Trispuffer lösen.

$\mathrm{Zu} 2 \mathrm{~m} l$ Trispuffer-DTNB-Lösung wurden zugesetzt

a) $0,02 \mathrm{ml}$ Serum

b) 0,2 mg PAM in $0,02 \mathrm{~m} /$ Trispuffer

c) $0,2 \mathrm{mg}$ Toxogonin in $0,02 \mathrm{ml}$ Trispuffer.

Es wurde einige Min. bei $22^{\circ}$ vorinkubiert, sodann die Reaktion durch Zusatz von $0,2 \mathrm{ml}$ Acetylthiocholinjodidlösung ( $55 \mathrm{~mm}$ ) gestartet.

Reaktionstemperatur $22^{\circ}$.

Gemessen wurde die Extinktionszunahme bei $405 \mathrm{~nm}$ in $1 \mathrm{~cm}$ Küvetten (siehe Abb. 1).

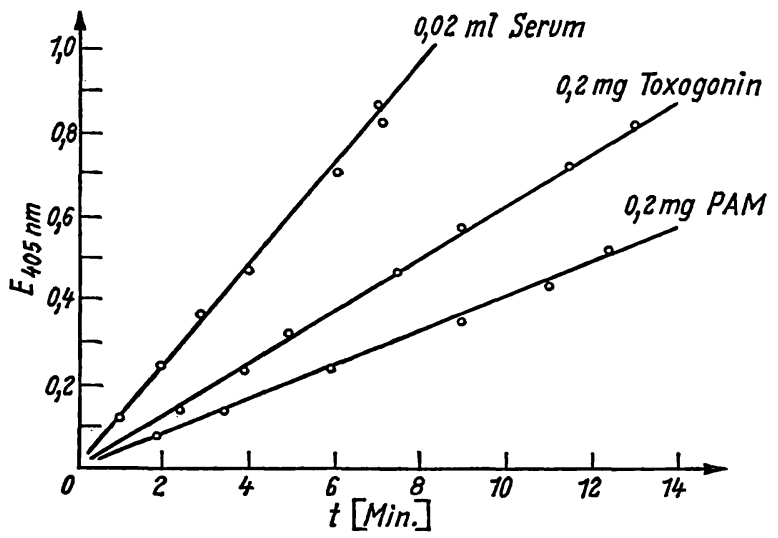

Abb. 1

Spaltung von Acetylthiocholin durch Serum, 2-PAM und Toxogonin

Für Acetylthiocholin wurde unter den genannten Bedingungen auch die Abhängigkeit der Substratspaltung von verschiedenen Oximmengen, nämlich 0,$05 ; 0,1 ; 0,2$ und $0,3 \mathrm{mg}$ Toxogonin sowie 0,$1 ; 0,2 ; 0,3$ und $0,4 \mathrm{mg}$ PAM pro Ansatz festgestellt (Abb. 2),

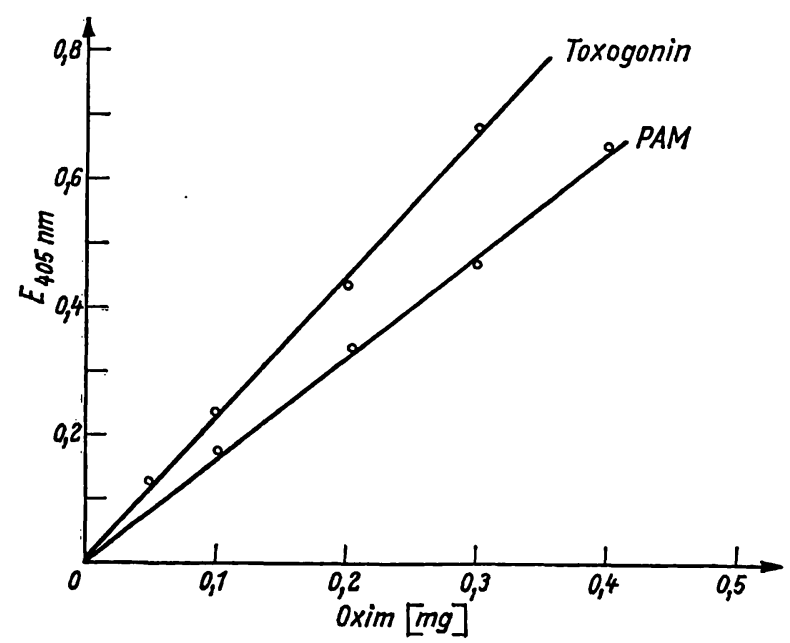

Abb. 2

Spaltung von Acetylthiocholin durch verschiedene Oximmengen. Gemessen wurde jewells die Extinktionszunahme innerhalb von 8 Min. 


\section{Buty'ylthiocbolinjodid}

Wir verwendeten den Reagenzsatz Merckotest; Cholinesterase, kinetischer Test ${ }^{1}$ ), der auf die Untersuchungen von KNEDEL und BÖTTGER (9) aufbaut.

Reaktionsbedingungen:

Endkonzentration im Ansatz vor der Bestimmung:

$0,02 \mathrm{M}$ Phosphatpuffer $\mathrm{pH} 7,7$

$6 \mathrm{~mm}$ S-Butyrylthiocholinjodid

0,25 mM 5,5-Dithiobis-2-nitrobenzoesäure.

$\mathrm{Zu} 2 \mathrm{~m} l$ der obigen Lösung wurden zugesetzt:

a) $0,01 \mathrm{~m} /$ Serum

b) $0,1 \mathrm{mg}$ PAM in $0,01 \mathrm{~m} l$ Phosphatpuffer

c) $0,1 \mathrm{mg}$ Toxogonin in $0,01 \mathrm{~m} /$ Phosphatpuffer.

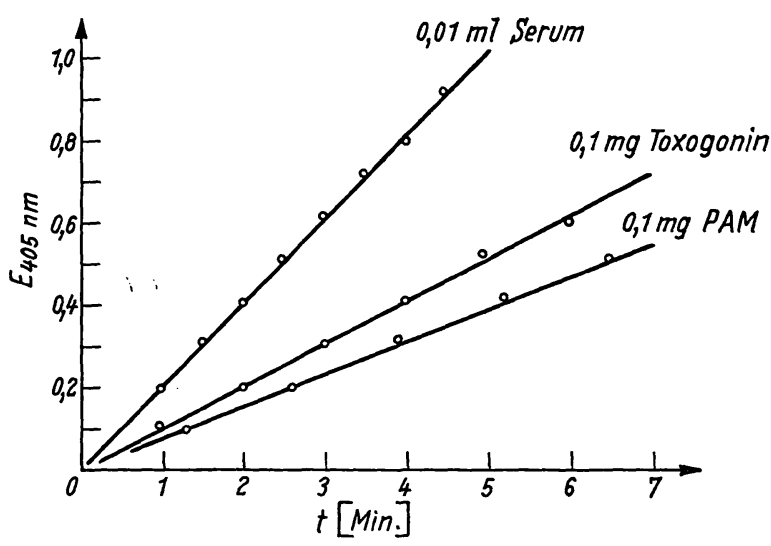

Abb. 3

Spaltung von Butyrylthiocholin durch Serum, 2-PAM und Toxogonin

\section{Reaktionstemperatur: $22^{\circ}$.}

Gemessen wurde die Extinktionszunahme bei $405 \mathrm{~nm}$, der starken Extinktionszunahme wegen abweichend von der Vorschrift in 0,5 cm-Küvetten (Abb. 3).

Für Butyrylthiocholin wurde unter den genannten Bedingungen auch die Abhängigkeit. der Substratspaltung von verschiedenen Oximmengen, nämlich 0,$1 ; 0,2$ und $0,3 \mathrm{mg}$ pro Ansatz, festgestellt (Abb. 4):

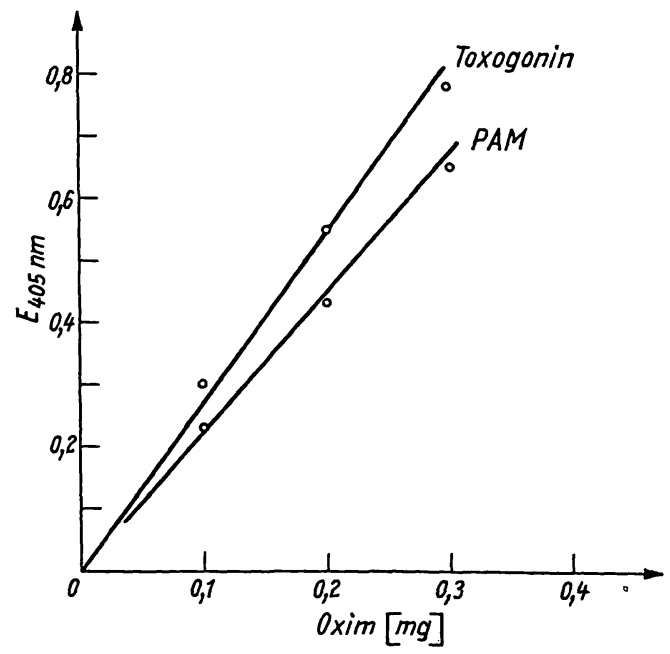

Abb. 4

Spaltung von Butyrylthiocholin durch verschiedene Oximmengen Gemessen wurde jeweils die Extinktionszunahme innerhalb von 3 Min.

1) Hersteller: E. Merck, Darmstadt.

\section{o-Nitrophenylbutyrat}

nach Main, MrLes und Braid (11).

Reaktionsbedingungen:

Butanol-Phosphatpuffer:

$5 \mathrm{ml}$ Butanol $+95 \mathrm{ml} 0,05 \mathrm{M}$ Phosphátpuffer $\mathrm{pH} 7,6$,

o-Nitrophenylbutyrat-Stammlösung:

$0,5 \mathrm{M}$ in Methanol.

Substrat-Pufferlösung (frisch herstellen):

$100 \mathrm{~m} l$ Butanol-Phosphatpuffer $+0,3 \mathrm{~m} l$ o-NitrophenylbutyratStammlösung.

$\mathrm{Zu} 5 \mathrm{~m} l$ Substrat-Pufferlösung wurden zugesetzt:

a) $0,004 \mathrm{~m} l$ Serum in $1 \mathrm{ml}$ Butanol-Phosphatpuffer

b) $0,3 \mathrm{mg}$ PAM in $1 \mathrm{~m} /$ Butanol-Phosphatpuffer

c) $0,3 \mathrm{mg}$ Toxogonin in $1 \mathrm{~m} /$ Butanol-Phosphatpuffer.

Reaktionstemperatur: $25^{\circ}$.

Gemessen wurde nach Zusatz von $0,05 \mathrm{ml}$ DFP (Diisopropylfluorphosphat)-Lösung $(0,025 \mathrm{M}$ in Isopropanol) und Auffüllen mit 50proz. Athanol ad 10,0 die Extinktionszunahme bei $414 \mathrm{~nm}$ in $1 \mathrm{~cm}-$ Küvetten (Abb. 5).

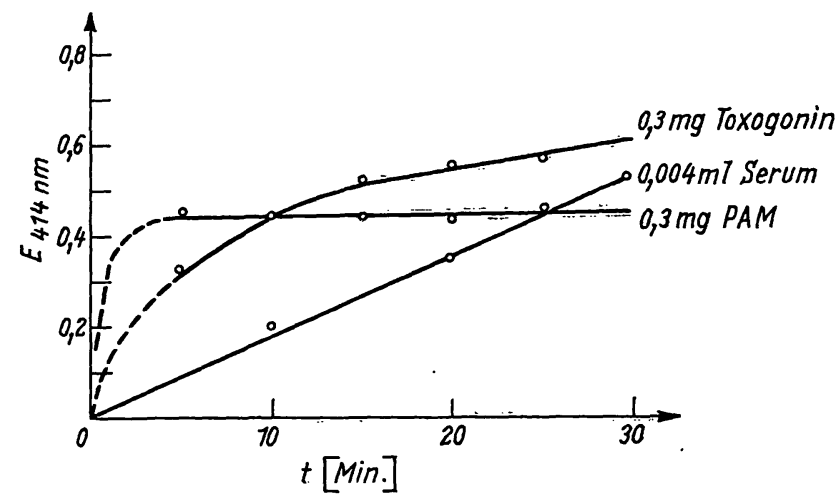

Abb. 5

Spaltung von o-Nitrophenylbutyrat durch Serum, 2-PAM und Toxogonin

Für o-Nitrophenylbutyrat wurde unter den genannten Bedingungen auch die Abhängigkeit der Substratspaltung von verschiedenen Oximmengen, nämlich 0,$1 ; 0,3 ; 0,4$ und $0,6 \mathrm{mg} 2-\mathrm{PAM}$ sowie 0,$1 ; 0,2 ; 0,3$ und $0,4 \mathrm{mg}$ Toxogonin pro Ansatz, festgestellt (Abb. 6):

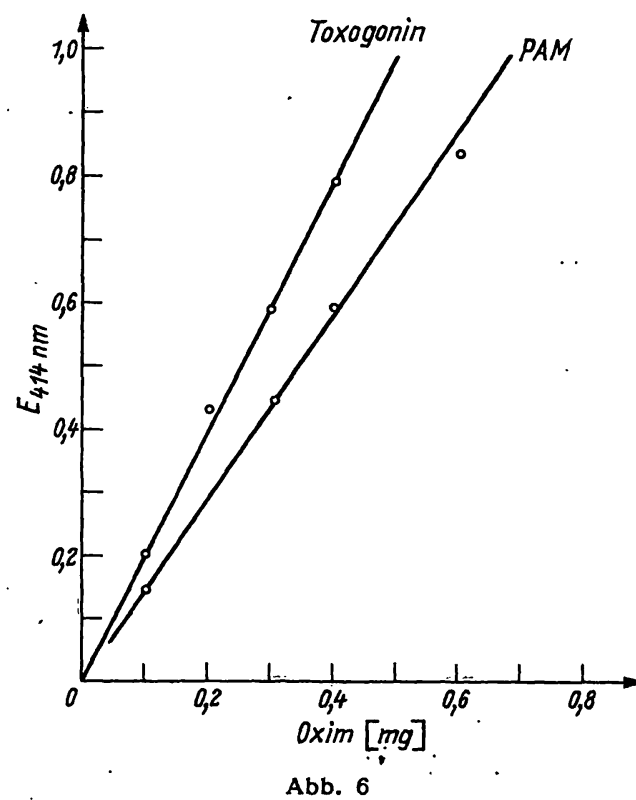

Spaltung vọn o-Nitrophenylbutyrat durch verschiedene Oximmengen innerhalb von 30 Min.

Z. klin. Chem, u. klin. Biochem. /.7. Jahrg. 1969 / Heft 5 


\section{Indoxylacetat}

nach URIEL (12)

Reaktionsbedingungen:

$5 \mathrm{mg}$ Indoxylacetat wurden in $0,5 \mathrm{ml}$ Aceton gelöst und sofort in $22 \mathrm{ml}$ Veronalpuffer $\mathrm{pH} 8,2 ; \mu=0,05$ gegeben.

$\mathrm{Zu} 2 \mathrm{ml}$ Puffer-Aceton-Substratgemisch wurden zugesetzt:

a) $0,1 \mathrm{~m} l$ Serum

b) $0,1 \mathrm{mg}$ PAM in $0,1 \mathrm{ml}$ Veronalpuffer

c) $0,1 \mathrm{mg}$ Toxogonin in $0,1 \mathrm{~m} l$ Veronalpuffer.

Reaktionstemperatur: $22^{\circ}$

Gemessen wurde die Extinktionszunahme bei $667 \mathrm{~nm}$ in $1 \mathrm{~cm}-$ Küvetten (Abb. 7):

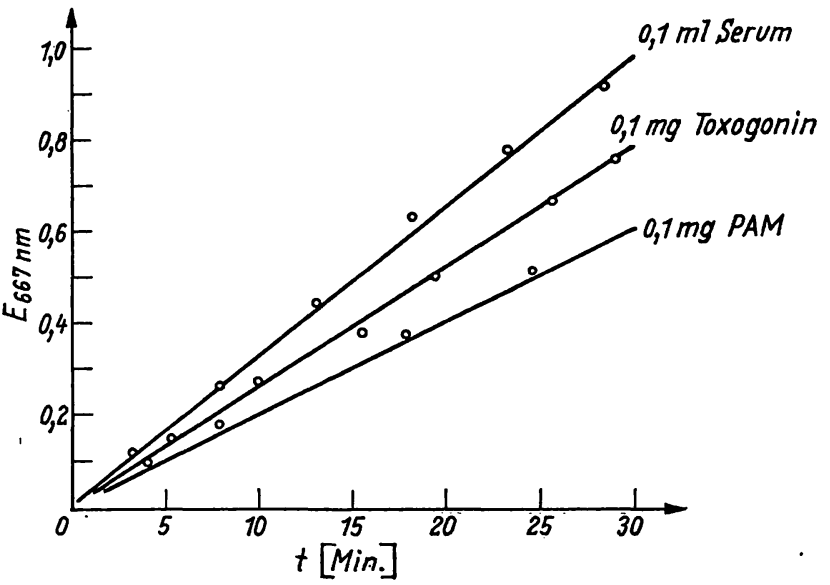

Abb. 7

Spaltung von Indoxylacetat durch Serum, 2-PAM und Toxogonin

Phenylbenzoat:

nach Rider, Moeller und DuBors (13).

Reaktionsbedingungen:

Phosphatpuffer: $5,35 \mathrm{~g} \mathrm{Na}_{2} \mathrm{HPO}_{4} \times 12 \mathrm{H}_{2} \mathrm{O}$ und $7 \mathrm{~g} \mathrm{NaH}_{2} \mathrm{PO}_{4} \times$ $\mathrm{H}_{2} \mathrm{O}$ wurden in Wasser ad 1000 gelöst.

Phenylbenzoat-Stammlösung: $2 \mathrm{~g}$ Phenylbenzoat in $100 \mathrm{~m} l$ Methanol.

Puffer-Substratlösung: $500 \mathrm{~m} l$ Phosphatpuffer $+1 \mathrm{ml}$ Phenylbenzoat-Stammlösung.

$\mathrm{Zu} 1 \mathrm{~m} l$ Puffer-Substratlösung wurden zugesetzt:

a) $1 \mathrm{~m} l$ Serum

b) $1 \mathrm{mg}$ PAM in $1 \mathrm{ml}$ Phosphatpuffer

c) $1 \mathrm{mg}$ Toxogonin in $1 \mathrm{~m} l$ Phosphatpuffer.

Reaktionstemperatur: $22^{\circ}$.

Gemessen wurde die Extinktionszunahme bei $360 \mathrm{~nm}$ in $1 \mathrm{~cm}$ Küvetten (Abb. 8):

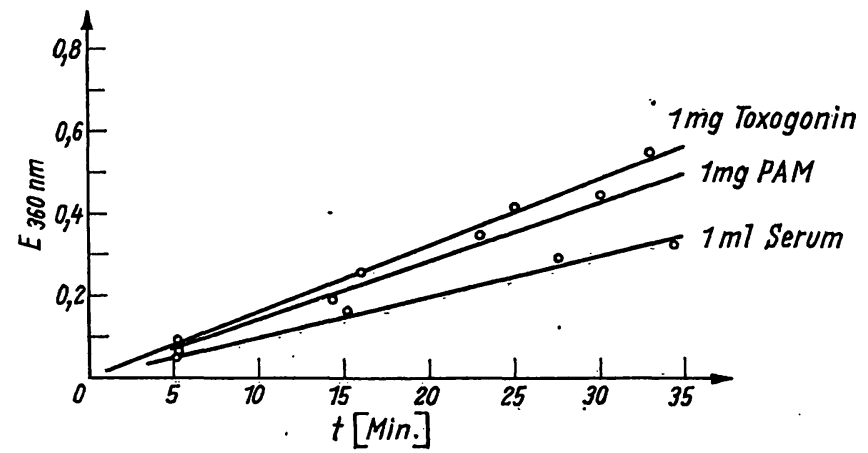

Abb. 8

Spaltung von Phenylbenzoat durch Serum, 2-PAM und Toxogonin

\section{$\alpha-N a p b t y l a c e t a t$}

Wir arbeiteten nach URIEL (12) unter Verwendung von $\alpha-\mathrm{Naph}$ tylacetat als Substrat und Echtblausalz B als Farbsalz.
Reaktionsbedingungen:

0,15M Phosphatpuffer: $\mathrm{pH}=7,2$.

$5 \mathrm{mg} \alpha$-Naphtylacetat wurden in $0,5 \mathrm{ml}$ Aceton gelöst und zusammen mit $1 \mathrm{mg}$ Echtblausalz $\mathrm{B}$ in $25 \mathrm{ml}$ Phosphatpuffer gegeben.

Zu $1 \mathrm{~m} l$ Puffer-Substrat-Farbsalzlösung wurden zugesctzt:

a) $0,1 \mathrm{~m} l$ Serum $+0,9 \mathrm{~m} l$ Phosphatpuffer

b) $1 \mathrm{mg} P A M$ in $1 \mathrm{~m} /$ Phosphatpuffer

c) $1 \mathrm{mg}$ Toxogonin in $1 \mathrm{~m} /$ Phosphatpuffer.

Reaktionstemperatur: $22^{\circ}$.

Messung der Extinktion in $1 \mathrm{~cm}-$ Küvetten.

In Ansatz a) mit Serum entwickelte sich bei der Inkubation ein gelbroter Farbton. Gemessen wurde die Extinktion im Absorptionsmaximum bei $515 \mathrm{~nm}$.

In Ansatz b) mit PAM lag das Absorptionsmaximum bei $535 \mathrm{~nm}$. Die Extinktionszunahme wurde hicr verfolgt. Sie war bei $515 \mathrm{~nm}$ jeweils etwas geringer.

Ansatz c) mit Toxogonin entwickelte einen blauroten Farbton. Das Absorptionsmaximum, in dem die Extinktionszunahme verfolgt wurde, lag bei $689 \mathrm{~nm}$. Daneben wird auch die sehr geringe Extinktionszunahme bei $515 \mathrm{~nm}$ angegeben (Abb. 9).

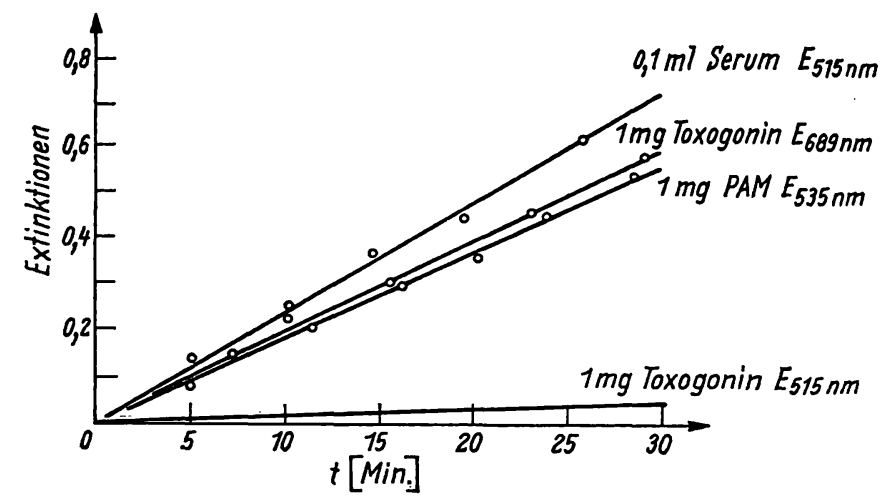

Abb. 9

Spaltung von $\alpha$-Naphtylacetat durch Serum, 2-PAM und Toxogonin

\section{Acetylcholin:}

nach FRIEDBERg und SAKAI (2) in der Warburg-Apparatur ${ }^{2}$ ).

Reaktionsbedingungen:

Lösungsmittel und Verdünnungsflüssigkeit für Acetylcholinchlorid, Serum, PAM und Toxogonin:

10 Teile 0,9proz. $\mathrm{NaCl}-\mathrm{Lösung}+3$ Teile 1,26proz. $\mathrm{NaHCO}_{3}$ Lösung.

Zur Einstellung eines pH-Wertes von etwa 7,73 (vgl. SüLLMranN (14)) wurde $1 / 2$ Std. mit einem Gasgemisch $\left(5 \% \mathrm{CO}_{2}, 95 \% \mathrm{~N}_{2}\right)$ gesättigt.

$\mathrm{Zu} 1,5 \mathrm{ml}$ einer 0,5 proz. Acetylcholinchloridlösung wurden zugesetzt:

a) $0,05 \mathrm{~m} l$ Serum $+0,45 \mathrm{ml}$ Verdünnungsflüssigkeit

b) $0,1 \mathrm{mg}$ bzw. $2 \mathrm{mg}$ PAM in $0,5 \mathrm{~m} l$ Verdünnungsflüssigkeit

c) $0,1 \mathrm{mg}$ bzw. $2 \mathrm{mg}$ Toxogonin in $0,5 \mathrm{~m} /$ Verdünnungsflüssigkeit. Reaktionstempexatur: $37^{\circ}$.

Gemessen wurden $\mu l \mathrm{CO}_{2} / \mathrm{Min}$.; Endablesung nach $60 \mathrm{Min}$ (Abb. 10).

\section{Butyrylcbolin}

Manometrische Bestimmung:

Reaktionsbedingungen wie bei Acetylcholin beschrieben, Butyrylcholinchlorid-Lösung 0,5proz., Serummenge pro Ansatz $0,02 \mathrm{ml}$.

Ergebnisse siehe Abbildung 11.

2) Wir danken der Firma Wacker, Burghausen, für die freundliche apparativie Unterstützung. 


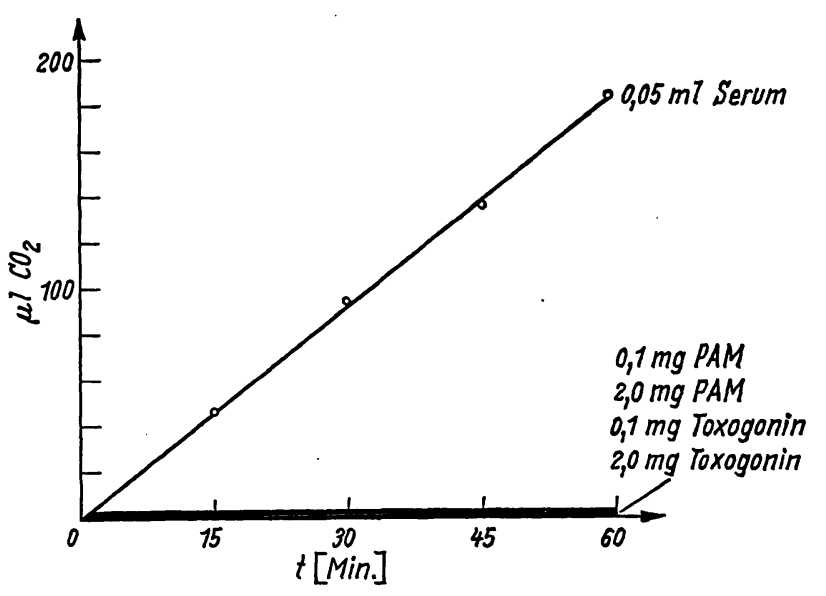

Abb. 10

Reaktion von Acetylcholin mit Serum, 2-PAM und Toxogonin

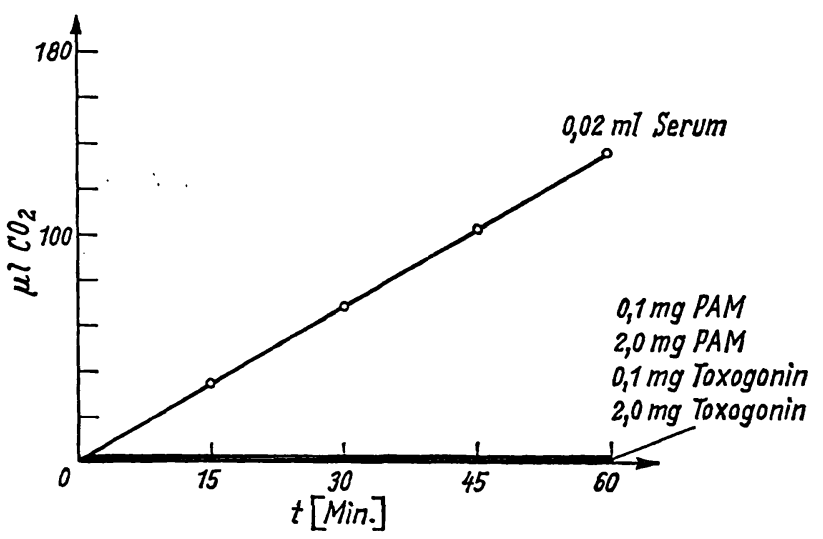

Abb. 11

Reaktion von Butyrylcholin mit Serum, 2-PAM und Toxogonin

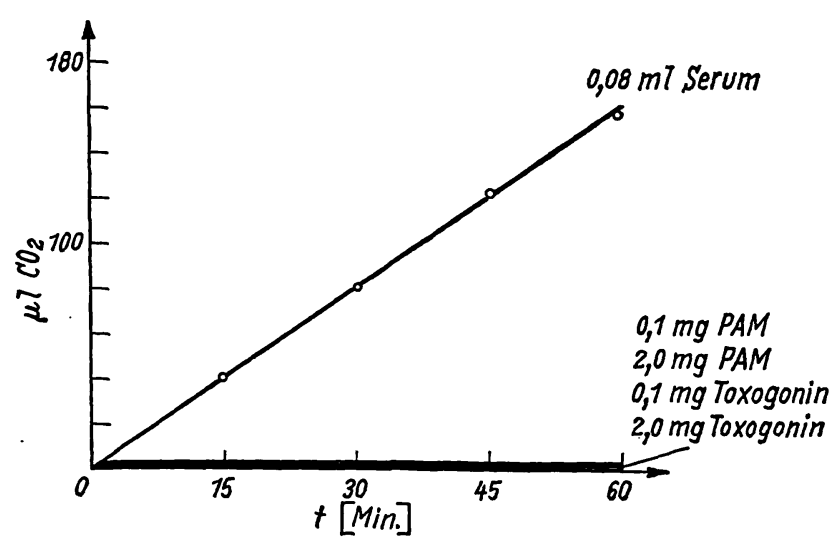

Abb. 12 Reaktion von Benzoylcholin mit Serum, 2-PAM und Toxogonin

\section{Benzoylcholin}

Manometrische Bestimmung:

Reaktionsbedingungen wie bei Acetylcholin angegeben unter Verwendung einer 0,15proz. Benzoylcholinchloridlösung und $0,08 \mathrm{ml}$ Serum pro Ansatz. Ergebnisse siehe Abbildung 12.

Der optische Test mit Messung der Extinktionsabnahme bei $240 \mathrm{~nm}$ (vgl. z. B. GoEDDE und ALtrand (15)) ist schon in Gegenwart geringer PAM- und Toxogoninmengen nicht anwendbar, da beide Oxime bei $240 \mathrm{~nm}$ stark absorbieren.

\section{Diskussion}

Unsere Versuchsergebnisse haben zunächst diejenigen von Bergner und O'Neill (5), Geldmacher-v. MaLIINCKRODT und Kaiser (7), HeitmanN und FelgeN-
HAUER (1) sowie NenNer und ERdmann (4) bestätigt, wonach Acetylthiocholin, Butyrylthiocholin, o-Nitrophenylbutyrat und $\alpha$-Naphthylacetat durch Oxime wie 2-PAM bzw. Toxogonin gespalten werden. Wir konnten weiter zeigen, $\mathrm{da} B$ auch Indoxylacetat und Phenylbenzoat in der gleichen Weise reagieren. Die Versuche wurden unter den von den einzelnen Autoren für die Substratspaltung durch Cholinesterase angegebenen Bedingungen durchgeführt. Die Oxime wurden den Ansätzen in Mengen zugesetzt, die etwa gleichen Umsatz ergaben wie die jeweils eingesetzte Serummenge. Eine Beziehung derart, daß eine bestimmte Oximmenge für alle Substrate den gleichen Umsatz ergab wie eine entsprechende Serummenge, ließ sich nicht ableiten.

Ergänzende, hier nicht im einzelnen wiedergegebene Versuche wurden auch mit E 600-vergiftetem Serum durchgeführt. Erfolgte die E 600-Zugabe so, daß eine Restaktivität von $10-20 \%$ verblieb, und wurde $8 \mathrm{Stdn}$. nach der Hemmstoffzugabe die Aktivität sowohl allein als auch nach Zusatz verschiedener 2-PAM- und Toxogoninmengen gemessen, so ergab sich eine Substratspaltung, die über der durch 2-PAM bzw. Toxogonin allein zu erreichenden lag, jedoch niedriger als die Summe der zu erwartenden Spaltung durch das Oxim und die verbliebene Restaktivität des Serums war. Es überlagern sich offenbar die reine Oximwirkung, die Hemmwirkung des Oxims auf die Cholinesterase und die einsetzende Reaktivierung des vergifteten Enzyms durch das Oxim.

Acetylcholin, Butyrylcholin und Benzoylcholin waren durch 2-PAM und Toxogonin in der genannten sowie in 20 fach höherer Konzentration, wie sie z. B. von FrIEDBERG und SAKAI zur in vitro-Reaktivierung alkylphosphatvergifteter Cholinesterasen empfohlen werden, nicht spaltbar.

Damit bestätigte sich für die untersuchten Sủbstrate die von GelDMACher-v. MaLIINCKRodt und KaISER (7) geäußerte Vermutung, daß nur echte Ester gegen Oxime stabil sind, während Substrate mit Säureanhydridcharakter, wie mehrfach für Oxim-ähnliche Verbindungen auch von anderen Autoren beobachtet (Literatur s. bei O'BriEN (16)), gespalten werden.

Wie schon HettmanN und Felgenhauder (1) zeigten, erfolgt diese Spaltung im allgemeinen zunächst in linearer Abhängigkeit von der Zeit. Nur im Fall des o-Nitrophenylbutyrat ist relativ rasch ein weitgehend stöchiometrischer Umsatz sowohl für 2-PAM (GELDMACHERv. MALLINCKRODT und Katser (7)) als auch Toxogonin (Nerrs (17)) festzustellen.

Die Substratspaltung durch die beiden Oxime verläuft, wie wir für Acetylthiocholin, Butyrylthiocholin und oNitrophenylbutyrat prüften, in dem untersuchten Bereich weitgehend in linearer $\mathrm{Abhängigkeit}$ von der Oximmenge. Dies zeigten auch NENNER und ERDMANN (4) für Acetylthiocholin und Toxogonin.

Um die Frage $z u$ beantworten, inwieweit die untersuchten, mit Oximen reagierènden Substrate für die Kontrolle einer in vivo-Reaktivierung der Serumcholinesterasen nach therapeutischen Gaben von 
Tab. 1

\begin{tabular}{|c|c|c|c|c|c|}
\hline \multirow[b]{2}{*}{ Autor } & \multirow[b]{2}{*}{ Substrat } & \multicolumn{2}{|c|}{ Reaktionsansatz } & \multicolumn{2}{|c|}{$\begin{array}{l}\text { Bei maximaler Blutkonz. in den } \\
\text { Ansatz gebracht nach Gabe von }\end{array}$} \\
\hline & & $\begin{array}{l}\text { zugesetzte } \\
\text { Serummenge }\end{array}$ & Endvolumen & $\begin{array}{l}\text { Toxogonin } \\
250 \mathrm{mg} \text { i. } \mathrm{v} \text {. }\end{array}$ & $\begin{array}{l}\text { 2-PAM } \\
500 \mathrm{mg} \mathrm{i.} \mathrm{v} .\end{array}$ \\
\hline $\begin{array}{l}\text { MAIN, MILES, BRAID (11) } \\
\text { KNEDEL und BöTTGER (9) } \\
\text { WEBER (10) }\end{array}$ & $\begin{array}{c}\text { o-Nitrophenylbutyrat } \\
\text { Butyrylthiocholin } \\
\text { Acetylthiocholin }\end{array}$ & $\begin{array}{l}0,004 \mathrm{ml} \\
0,01 \mathrm{ml} \\
0,02 \mathrm{ml}\end{array}$ & $\begin{array}{l}6 \mathrm{ml} \\
2 \mathrm{ml} \\
2 \mathrm{ml}\end{array}$ & $\begin{array}{l}0,2 \mu \mathrm{g} \\
0,5 \mu \mathrm{g} \\
1,0 \mu \mathrm{g}\end{array}$ & $\begin{array}{l}0,4 \mu \mathrm{g} \\
1,0 \mu \mathrm{g} \\
2,0 \mu \mathrm{g}\end{array}$ \\
\hline
\end{tabular}

2-PAM und Toxogonin geeignet sind, müssen die im Reaktionsansatz enthaltene Serummenge und die Oximkonzentration im Serum berücksichtigt werden. Die Therapie mit 2-PAM und Toxogonin erfolgt im allgemeinen durch i.v.-Injektion. Würde unmittelbar darauf Blut entnommen, so wäre bei Gabe von $500 \mathrm{mg}$ 2-PAM bzw. $250 \mathrm{mg}$ Toxogonin mit einer maximalen Serumkonzentration von $100 \mu \mathrm{g} / \mathrm{m} l$ für $2-\mathrm{PAM}$ bzw. $50 \mu \mathrm{g} / \mathrm{m} l$ für Toxogonin zu rechnen. Mit zunehmendem Abstand zwischen einmaliger Injektion und Blutentnahme nimmt diese Konzentration entsprechend der Halbwertszeit der Oxime im Blut (etwa 2 Std. für 2-PAM-jodid nach KoNDRITZER und Mitarbeiter (18) sowie für Toxogonin nach ERdmanN, Bosse und Franke (19)) ab.

CALESNICK und Mitarbeiter (20) fanden 30 Min. nach i.v.-Injektion von $45 \mathrm{mg} 2-\mathrm{PAM}-\mathrm{Cl} / \mathrm{kg}$ beim Menschen einen Blutspiegel von $29,3 \mu \mathrm{g} / \mathrm{m} l$.

Legt man diese Werte zugrunde, so können bei Bestimmung der Serum-Cholinesterase-Aktivität mit den wichtigsten der genannten Verfahren dem Reaktionsansatz nach einmaliger Injektion der üblichen therapeutischen Dosis durch die eingesetzte Serummenge maximal 2-PAM und Toxogonin entsprechend der Tabelle 1 zugeführt sein.

Die in den einzelnen Reaktionsansätzen enthaltenen Oximmengen betragen demnach höchstens $1 / 50$ der von uns zur Prüfung eingesetzten Mengen. Sie bedingen damit eine Substratspaltung, die höchstens den entsprechenden Anteil der durch normales Serum verursachten Substratspaltung ausmacht. Daraus ergibt sich, da $B$ bei Entnahme von Serum nach einmaligen therapeutischen 2-PAM- und Toxogoningaben in der angegebenen Dosierung zur Prüfung auf eine eingetretene Reaktivierung der Serum-Cholinesterasen mit den drei eben genannten
Verfahren keine erhebliche Verfälschung des Befundes eintritt. Nur mehrfache, relativ kurz hintereinander erfolgende Oxim-Injektionen könnten auf Grund eines durch Kumulierung höheren Blut-Oximspiegels zu fehlerhaften Ergebnissen führen. Bei anderen Verfahren die mit größeren Serummengen arbeiten, ist die Fehlermöglichkeit entsprechend größer.

Für Reaktivierungsversuche in vitro, für welche 2-PAM-Konzentrationen im Bereich von $10^{-3}$ molar empfohlen werden (FrIEDberG und SAKaI (2)), können dagegen als Substrate bisher nur echte Ester wie Acetylcholin, Butyrylcholin und Benzoylcholin als brauchbar angesehen werden.

Bei der Beurteilung der erhobenen Befunde ist daran zu denken, daßder fehlende Einfluß einer Oximtherapie oder eines Oximzusatzes in vitro auf alkylphosphatvergiftete Serumcholinesterasen nicht unbedingt einen therapeutischen Effekt ausschließen muß, da für die Giftwirkung von Alkylphosphaten auf den Organismus die Hemmung der Organ-Cholinesterasen ausschlaggebend sein dürfte, die ein anderes Verhalten als das Serumenzym zeigen können.

Zieht man aus diesem Grunde, wie von NenNer und ERDMANN (4) angeregt, zur Verlaufskontrolle die Aktivität der Acetylcholinesterase der Erythrocyten mit heran, so gelten auch hier für die Substratauswahl die genannten Gesichtspunkte. Entsprechendes ist bei histochemischen Untersuchungen zu berücksichtigen.

Die Arbeit wurde mit Hilfe der Deutschen Forschungsgemeinschaft durchgeführt, wofür wir auch an dieser Stelle unseren besten Dank aussprechen.

Die Präparate 2-PAM-Jodid und Toxogonin wurden uns liebenswürdigerweise von Herrn Dr. LORKe, Leiter des Instituts für Toxikologie der Farbenfabriken Bayer AG, zur Verfügung gestellt, wofür wir herzlich danken.

\section{Literatur}

1. Heitmann, R. und K. Felgenhauer, Dtsch: Med. Wschr. 94, 224 (1969). - 2. Friedberg, K. D. und F. SAKAr, Dtsch. Zschr. gerichtl. Med. 47, 580 (1958). - 3. Ammon, H., Pflügers Arch. Physiol. 233, 486 (1934). - 4. NenNer, M. und W. D. ERdManN, Dtsch. Med. Wschr. 94, 504 (1969). - 5. BERGNER, A. D., und J. J. O'NeIlL, J. Histochem. Cytochem. 6, 72 (1958). - 6. HeILBRONN, E., Acta Chem. Scand. 13, 1547 (1959). - 7. GELdMACHERv. Mallinckrodt, M. und I. Kaịsér, diese Z. 6, 141 (1968). 8. SuCKER, H., Enzymatische Methoden, in: Gadamers Lehrbuch der chemischen Toxikologie und Anleitung zur Ausmittelung der Gifte, Bd. II, Hrsg. E. Graf und Fr. R. Preuss. Van den Hoek und Rupprecht, Göttingen (1966). - 9. KNEDEL, .M. und R. Bötrger, Klin. Wschr. 45, 325 (1967). - 10. Weber, H., Dtsch. Med. Wschr. 91, 1927 (1966). - 11. Marn, A. R., K. E. Mrles und P. E. Braid, Biochem. J. 78,769 (1961). - 12. URreL, J., Ann. Inst. Pasteur 101, 104 (1961). - 13. Rider, J. A., H. C. Mozller, und K. P. DuBors, Proc. Soc. Exp. Biol. Med. 76,
427 (1951). - 14. SülLMANN, H., Manometrische Methoden zur Untersuchung des Gewebsstoffwechsels. in: Handbuch der Physiologisch-Pathologisch-Chemischen Analyse, Hrsg. K. Lang und E. Lehnartz, Bd. VI/A Enzyme, Teil A Springer, Berlin, Heidelberg (1964). - 15. GozdDe, H. W. und Altuand, K., Arbeitsvorschriften. in: Goedde, H. W., Doenicke, A. und Altuand, K., Pseudocholinesterasen. Springer-Verlag Berlin, Heidelberg, New York (1967). - 16. O'BrIEN, R. D., Toxic phosphorus esters. Academic Press New York und London (1960). - 17. NewLs, M., Dissertation Erlangen, in Vorbereitung. - 18. KonDRITZER, A. A., P. Zvirblis, A. Goodman und S. H. Paplanus, J. Pharm. Sci. 57, 1142 (1968). - 19. ERdmanN, W. D., I. Bosse und P. Franke, Dtsch. Med. Wschr. 90, 1436, (1965). - 20. Calesnick, B., J. A. Christensen, und M. Richter, Arch. Environ. Health 15, 599 (1967).

Priv.-Doz. Dr. Dr. Marika Geldmacher-von Mallinckrodt 8520 Erlangen, Universitätsstraße 22 